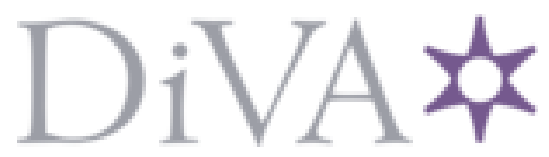

http://www.diva-portal.org

This is the published version of a chapter published in Higher creativity for virtual teams - Developing Platforms for Co-Creation.

Citation for the original published chapter:

Larsson, A., Larsson, T., Bylund, N., Isaksson, O. (2007)

Rethinking virtual teams for streamlined development.

In: Higher creativity for virtual teams - Developing Platforms for Co-Creation (pp. 138-156).

Hershey, PA: Information Science Reference

N.B. When citing this work, cite the original published chapter.

Permanent link to this version:

http:// urn.kb.se/resolve?urn=urn:nbn:se:bth-11282 


\title{
Rethinking Virtual Teams for Streamlined Development
}

\author{
Andreas Larsson, Luleå University of Technology, Sweden \\ Tobias Larsson, Luleå University of Technology, Sweden \\ Nicklas Bylund, Volvo Car Corporation, Sweden \\ Ola Isaksson, Volvo Aero Corporation, Sweden
}

\section{Introduction}

The motivation for this chapter is to question, or at least provide a reality check on some of the 'quick fix recipes' for virtual team creativity that often seem to take a rather idealised perspective on how creativity might be infused in geographically dispersed development teams. Our stance towards creativity techniques is generally positive. Not surprisingly, we too believe that creativity is a crucial building block of successful product development in just about any industry context. However, much of the research on creative teams tends to focus mainly on relatively small teams working in the very front-end of product development, such as in the initial idea generation phase of a consumer electronics project. If anything, such development projects explicitly target the creative aspects of collaboration to showcase innovative concepts for a future market. We bring a complementary perspective from an industry context where creativity is often perceived as risky business - yet a precondition for success.

Consequently, we direct our focus to what we have chosen to call 'streamlined development' - here defined as development activities which are considered as relatively mature with respect to achieving accelerated development and significant cost reduction, while maintaining or increasing product performance. We do not imply through this term that the 'fuzzy front-end' of innovation is entirely free from harsh deadlines, demanding industry regulations, downstream production planning, or supply chain integration issues. We merely want to focus more closely on the concerns of virtual teams that might not usually describe their own work to be of a primarily 'creative' nature. First and foremost, we are interested in the individuals and teams that enter the field after the contracts have been written; the people whose job it is to 'deliver what has been promised'. Not only do we realise that these teams are in serious need of appropriate creativity enablers, we also realise that these teams currently work under circumstances where traditional approaches for enhancing creativity might no longer be satisfactory.

Drawing from experiences in highly streamlined product development work in the automotive and aerospace industries, we aim to provide insights into the complex relationships between geographically dispersed team members and the day-to-day activities through which they collectively create the cars and aero engines of tomorrow. The overall business context in these industries is rapidly and continuously changing, which has serious implications on how global partnerships are formed and sustained. For the virtual teams engaged in such partnerships, creativity is undoubtedly a key to success. However, as this chapter will highlight, we argue that it is time to radically progress our current understanding of how creativity could be introduced in organisations where factors like legal demands and contractual agreements severely restrict 'outside-the-box' thinking, and where well-known creativity enablers such as trust, shared goals, and shared culture are becoming increasingly difficult to accomplish. 
Some overall characteristics of automotive and aerospace development in a global perspective are elaborated upon using examples from two Swedish companies that have already taken significant steps towards realising the Virtual Enterprise vision. This chapter's 'tales from the battlefield' are also taken from these companies and are based on observations and experiences from two car platform development projects at Volvo Car Corporation, and from two aero engine development projects at Volvo Aero Corporation. These tales are thematically organised around four different dilemmas, which fundamentally implies that while establishing trust, shared goals, etc. seems to be highly important for successful collaboration in virtual teams, the special conditions under which these virtual teams work are often not conducive to building those creativity enabling properties. Furthermore, these dilemmas also imply that, for example, trust as a creativity enabler is not about an unconditional either/or question. Briefly stated, it is about knowing when to, and subsequently when not to, trust someone or a piece of information.

\section{Corporate Contexts}

Today, globalisation is a reality and not simply a matter of choosing whether or not to make use of worldwide production facilities and geographically dispersed product development teams. Considering this increasing 'virtualisation' of companies, an overview of the corporate contexts in which our forthcoming 'tales from the battlefield' are embedded is provided below. We initially describe some characteristics of automotive development, a business-toconsumer industry, where brands join forces to develop common platforms to reach scale advantages. Descriptions follow of some characteristics of aero engine development, a business-to-business industry, where component manufacturers create solutions in very close partnerships with several engine manufacturers (who are essentially fierce competitors).

\section{Automotive Platform Development}

The product life cycle in the automotive industry has been reduced to produce more and more models in a shorter time span. A typical life span of a car model is five to seven years. However, manufacturing techniques still rely heavily on a billion euro investment for tooling and assembly equipment, and the development cost is hundreds of millions of Euros. To amortise the investments and development costs, companies are seeking scale advantages. If several automotive brands use the same platform, the volume of shared parts increases, meaning less customised manufacturing equipment, etc.

In the last 10 years, more and more automotive groups have been created. Very few independent manufacturers of size, such as BMW and Porsche, now exist. However, the idea of forming automotive groups is not new. For example, Chrysler Corporation joined the brands Chrysler, Dodge, Plymouth and DeSoto already in 1928, and then merged with Daimler-Benz to form DaimlerChrysler in 1998. General Motors' (Chevrolet, Pontiac, GMC, Saturn, Hummer, SAAB and Cadillac) history of mergers dates back to 1899, when Olds Motor Vehicle and Olds Gasoline Engine Works merged to form Olds Motor Works.

Note that an automotive platform does not just mean that some kind of go-cart like structure is made and different 'top hats' are put on. Although it is true that the lower part of the car body and the suspension are important parts in a platform, there are also important synergy and scale effects regarding commodities, such as tyres, batteries, fasteners, paint, sealers, electronics, electrics, as well as more complex systems such as steering servos, anti-lock brake systems (ABS), air bags, and climate systems. Using engine and gearbox units in 
different cars on the same platform is also attractive because of the high development cost. There are even automotive groups, such as PSA, that develop engines to be used by other automotive groups.

To amortise investments and development costs over a longer period, the life of a platform for a car can be extended to cover several car models. This is especially valuable if the base program platform has shown its strength in successfully being used in a variety of cars for several car brands in an automotive group, e.g. station wagons, sedans, SUVs, convertibles, and minivans. New legal demands and higher customer expectations on the next generation of cars, together with increased competition lead to a situation where higher performance is needed at a lower or maintained price. The upgrade must start in time so that new models are ready to replace the old ones at the end of their designated life cycle. Based on data from all platform models, which come from different brands on the platform, a discussion is held about the possibilities and hindrances for upgrading the platform. Every brand has its profile, identity and customer segment meaning different priorities. Since the budget is limited and fixed and will most likely be reduced later on, it is necessary to know how the upgrades, i.e. the changed requirements, impact the cost. It is about balancing the content of the upgrade package. The whole procedure of agreeing on the requirement levels and basic solutions are made within both co-located and virtual teams, and enabling these teams to collaborate more effectively is undoubtedly a key priority for all participating brands.

\section{Aero Engine Development}

The aerospace industry provides another example of an area where there is pressure to perform the product development on a global arena with many partners representing different companies - yet with a common business interest. Since development in commercial engine programs follows contractual agreements and the customers are professional organisations (i.e. airliners) rather than the actual users of transport services (i.e. passengers) - the lead time pressure will always be there. Once the airliners have placed their orders for a new aircraft and selected the engines, the development race starts.

It is important to recognize the huge program coordination required to certify new aircraft and their engines. In practical terms - there are some rather hard targets for all involved development teams to meet. From a technical perspective an aero engine is a thermodynamic machine, most often a gas turbine. The trade off is to balance technical system performance (weight, thrust, noise, and emissions etc.), durability and safety with the cost. While the system performance is designed and 'owned' primarily by the OEM (Original Equipment Manufacturer), the development of the engine components involves several different companies sharing both technical and financial risk (and also revenue, of course). Setting up a complete set of technical requirements sufficient for component design from day one is difficult, thus close collaboration with those partner companies is a must.

Another trend of the aerospace industry is that because the cost of development is increasing, and lead-time pressure is continuous, the 'window' for design and engineering work shortens. As a reference, the lead-time from signed contract when the actual development is launched to FETT (First Engine to Test) decreases about six to seven percent annually. Currently, development lead time is less than one and a half years. The time available for product development consequently decreases while, essentially, the same amount of decisions has to be taken. This is an immense challenge for any design team and is exacerbated by the challenges of collaborating effectively between different sites and different organisations. The design teams must reach peak performance each time, and failure to do so cause costly 
solutions that affect the entire life cycle of the engine in operation (about 40 years). The opportunity to modify already certified designs is limited due to the extensive certification process required for air worthiness.

Consequently, there are many incentives and drivers to more effectively enable the development teams to perform at their absolute best. We will discuss later situations that illustrate some of the challenges experienced in two recent engine development programs.

\section{Tales from the Battlefield}

In this section, we have chosen to emphasize the so called 'live experience' of engineers who work under the conditions described above; the engineers who are called to the 'battlefield' on a day-to-day basis and have to submit to the rules, regulations, procedures and routines that govern their work in the global product development projects in which they partake. These tales are based on experiences and observations from global product development projects carried out in the automotive and aeronautical industries, and we note that even though Volvo Car Corporation and Volvo Aero Corporation provide the primary source of information here, we have also collected insights from complementary sources within these global partnerships. It is not our intention to promote any kind of 'us vs. them' analytical lens and the examples we provide are thus not intended as examples of good or bad practice, rather our objective is to tell it as perceived by members of these global teams, that is, we discuss 'real' problems that engineers encounter while trying to get the job done. We neither give both sides of the coin, nor do we judge which corporate culture is 'best'. We merely offer you the opportunity to get some insight into the kinds of barriers to collaboration one could expect if embarking on global, boundary-crossing product development projects in streamlined industry domains.

First, we reconnect to the earlier description of the two main industry contexts, or 'battlefields' if you will, that we are dealing with: automotive platform development and aero engine development. Then, we have organized the tales from the battlefield according to four dilemmas. The reason for doing so is mainly that that we need to seriously reconsider some of the 'creativity enablers' that have, more or less, come to be taken for granted when discussing successful and creative virtual teams. Having said that, we do not imply that these wellknown enablers are entirely useless - on the contrary, we applaud just about any initiative to enable creativity in virtual teams. All we are saying is that there are particular circumstances of so called streamlined development that increase the complexity of global business partnerships, and which create barriers to creativity that are not easily forced. We would like to point out that the chosen dilemmas should not be considered as the only interesting aspects in this field, but as a selected set of issues that have been particularly prominent in the settings that we have participated in and studied. Also, we are using the term 'dilemma' rather loosely, to signify perplex situations that require making choices between equally unfavourable options, such as choosing between risking a joint partnership to secure individual rewards, or risking the individual rewards to reap potential future benefits of a joint partnership.

To illustrate our point, we make reference to Sutton's (2001) basic principles for differentiating between 'innovative work' and 'routine work' (see Table 1). As Sutton (2001) notes, both principles are necessary for moving forward, and the real challenge is thus not to choose between the two columns, once and for all, but rather to know when it is beneficial to exploit old ways and when it is time to explore new ways. 


\begin{tabular}{ll}
\hline Exploiting Old Ways: & Exploring New Ways: \\
Organizing for Routine Work & Organizing for Innovative Work \\
\hline Drive out variance & Enhance variance \\
See old things in old ways & See old things in new ways \\
Replicate the past & Break from the past \\
Goal: Make money now & Goal: Make money later \\
\hline
\end{tabular}

Table 1. Basic Organizing Principles: Exploitation vs. Exploration. (Sutton, 2001, p. 7)

With the below observations from the battlefield, we want to highlight that we are looking at global business partnerships where several fundamental pillars of a creative environment, at least according to conventional wisdom, seem to be missing (or at least seem extremely difficult to achieve), and yet these partnerships would cease to exist without creativity. Also, we are looking at situations where there are specific constraints that essentially force companies towards the left column of the above table - requiring that they efficiently manage to both replicate past successes and to know when to break completely new ground, thus moving to the right-hand column of Table 1.

\section{Battlefield A: Automotive Platform Development}

\section{Project A1: Compact Car Platform}

Several years ago, a medium-sized platform was developed at Ford Motor Company (FMC), including brands in Europe and Asia. The platform has proved successful and all three brands have made profit on vehicles based on the platform. Recently, a decision was made to update this platform to meet new legal requirements and new customer expectations. This process is essentially about defining improvement actions at minimum cost with respect to sourcing (i.e. finding the right supplier at maximum advantage), engineering and factory changes at the different brands. This is a fierce fight about getting the most from the platform and it is complicated by the different strategies of the constituent brands - a low cost brand will naturally have very different priorities from a premium brand. When this update started, some resident engineers were installed, as leaders, in order to 'fight for the brand' requirements. Since the brand that has the earliest production date locks the updated platform capability, it is important to be active already at the beginning even if the start of production is far in the future.

\section{Project A2: Full-size Car Platform}

This project is shared among three European brands within FMC and aimed to develop next generation full-size cars of different types. Here, one of the smaller companies within FMC has the lead of the platform - meaning that it is responsible for the 'orchestration'. In order to do this, the smaller company has needed to adapt to FMC's systems and routines, which has not been an easy feat. Early mistakes in the documentation and lack of understanding of the importance of formal agreements within FMC have lead to a lot of frustration and extra work. In FMC, a platform is 'remote controlled' by the documents, while in other brands, the development is often made more directly.

\section{Battlefield B: Aero Engine Development}

\section{Project B1: Large Commercial Jet Engine for an Airbus Aircraft}

A few years ago, when the engine for the new Airbus 'super jumbo' (A380) was in the development stages, Volvo Aero was responsible for the development of an integral, mechanical structure in the engine, and several other companies had similar responsibilities 
for interfacing components in the engine. Once the contract was signed, the team was set up to start the development work. Until then, the OEM had been responsible for pre-development but now the time had come to define the component in detail. The technical challenge was obvious, since the engine included many geometrical interfaces, and a complex geometry requires advanced castings in advanced materials (which consequently results in long lead time). The interaction with neighbouring development teams and their design models, and with the engine system level needed particular attention. These factors impacted on the decision to co-locate the Volvo Aero design team with their colleagues at the OEM site, and to support that remote team with back-office resources.

All involved were experienced in collaborating, highly dedicated, and aware of the importance of co-location to succeed. Ideally, the selection of such a team should be carefully planned, powered by training, team building, and IT tools for engineering and communication before 'take off'. Here is where reality strikes back. The first technical decision review gate was only a couple of weeks ahead. The conceptual design had been made by another team at the other company and needed to be understood quickly. A coordinator was sent to the 'hot zone'. The hunt for team members able to commit to staying abroad for a long period (starting immediately) was intense and the team on site was soon under intense pressure. The effort to support the team from back-office was also in start-up mode, which took time. As a consequence, the team on site needed to deal with more issues than originally planned.

\section{Project B2: Large Commercial Jet Engine for a Boeing Aircraft}

This project is more recent, and deals with the development of structural components for an engine that will be used on, for instance, the 787 Dreamliner from Boeing. The technical preconditions were quite similar to Project B1, with several partners co-developing the engine together with the integrator (the OEM). When the design team was set up, the plan was to quickly co-locate the core team at the Volvo Aero office in Sweden, mainly in order to better use routines and the more sparsely used resources outside the core team. Coordination and collaboration with the engine system level of design, as well as other design teams (from other companies) working on other components and sub-systems in the engine, had to be facilitated by other means than co-location.

The collaboration between the design teams, co-ordinated by the integrator, relied to a much higher degree on e-tools for collaboration, such as sharing meeting documents in virtual meetings. Also, several applications allowed Volvo Aero to remotely access tools and resources at the OEM. After a few initial weeks, Volvo Aero only needed to keep a representative on site, whose main task was to facilitate and coordinate communication between the design team in Sweden and the integrator. Figure 2 illustrates the difference in collaborative set-up between Project B1 and Project B2.
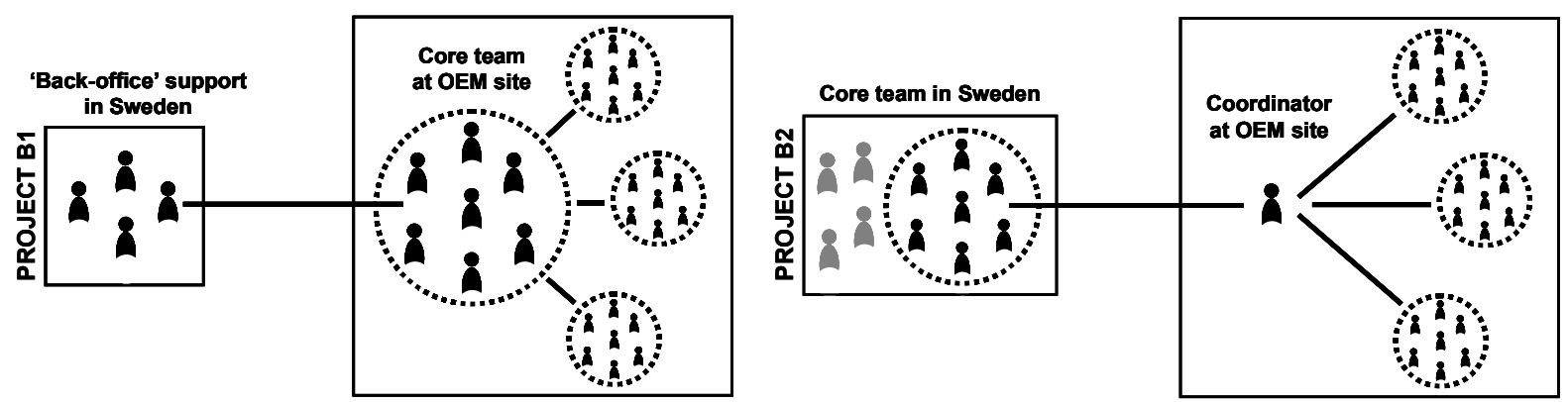

Figure 1. Comparison between Project B1 (left) and Project B2 (right). 


\section{Dilemma I: Trust}

The dilemma of trust is, in basic terms, that you need trust to develop trust, and to be trusted you must also trust others. The most important thing is that the concept applies only to situations where there exists some degree of uncertainty or risk. To a certain extent, Virtual Enterprises are about sharing risks and managing uncertainties in highly competitive markets.

Davidow and Malone (1992) note: "To achieve true partnership, customers and suppliers must share information - on new products, designs, internal business plans, and long-term strategy - that once was closely guarded" (p. 145). Although we are in general agreement with this statement, we would like to take the notion of true partnership a bit further. In our opinion, trust is not created once and for all; it is a highly context-dependent concept and thus changes over time. As we will highlight with examples below, it is crucial to know when to share and, subsequently, when not to share information or knowledge. In streamlined business areas, it is absolutely critical to continuously assess advantages and drawbacks on the individual, team, company, and virtual enterprise levels. In the case of automotive development, what is best for one brand has to be considered in relation to what is best for the common platform. In the case of aero engine development, what is best for one component must be related to what is best for the overall system.

It seems like we sometimes take incentives for collaboration too seriously and automatically assume that just because you may have a joint business offer you are eager to collaborate and will do everything in your power to make the relationship work as effectively and smoothly as possible. We think this is a too simplified view on the situation that many global companies face. Relating to the concept of 'coopetition' (Brandenburger \& Nalebuff, 1997), you have to collaborate to survive, but you also have to protect your own brand and expertise in order to stay competitive and to be perceived as a preferred partner in the future. Companies are struggling to know when, where and how to draw the line. As one project leader at Volvo Aero commented on the close relationship with three major engine companies (General Electric, Pratt \& Whitney, and Rolls-Royce):

"We never share anything which could harm these three companies. If we fall into disfavour with them it is a catastrophe for us. We never present evidence from [a company's] engine program to a competitor in order to come to a solution...we'd rather back off and find another way of proving our case."

If we consider trust to be a cornerstone of creativity, we must seriously think about the boundaries of such trust. In the above situation, the ability to establish mutual trust and respect is absolutely critical to set the stage for creative collaboration. Surely, these teams and companies need to trust each other to some extent in order to achieve the contract they have jointly agreed to, but in fulfilling their contract obligations, they should not be allowed to be 'too creative' or 'too trusting', thus risking to spoil future contracts, possibly with another partner. So, from a Virtual Enterprise perspective, with whom are you allowed to be really creative with, and with whom is it merely 'business-as-usual'? In a discussion about how creativity plays out in global aero engine alliances, one team member commented:

\section{"Creativity mostly happens in local teams, but sometimes also in distributed teams... but then you have to think about how you expose yourself."}

In automotive development, similar problems are faced in terms of staying loyal to the brand or staying loyal to the automotive group. Here, trust has a lot to do with the expectations of 
what each partner has to contribute to the common platform. Partly, this is a financial issue where the automotive group is calculating the sales volumes for each brand and the total project cost, which is then shared (in relation to expected sales) between platform partners. This means that there is a lot of documentation describing what each brand should deliver for a certain amount of money. It is thus absolutely crucial to keep tabs on what development is 'brand unique' (used only by one brand) and what is 'platform-wide' (used by all platform brands). For instance, there could be reinforcements to the platform floor, which are only needed by one brand. There are even parts that should be developed by the platform project, but which will only be used by one brand. Naturally, documents and contracts are highly important in such work to avoid bias.

"The nice form of creativity...figuring something out together...this is more a paper war. It is about knowing that on page six-hundred-and-twenty-two in the thousand-page document it says 'this is what the platform is doing, this is what the brand is doing'."

Another aspect that complicates the whole trust and bias discussion is that each brand has the responsibility for one platform size. This means that the brand that has 'lead' on a certain platform takes on a dual role: as platform leader and as brand representative in the platform project. This also impacts individual team members who have to move as seamlessly as they can between 'platform thinking' and 'brand thinking'. A similar situation arises in aero engine development, when Volvo Aero team members need to move between 'component thinking' (i.e. loyalty to own brand) and 'system thinking' (i.e. loyalty to engine manufacturers, aircraft manufacturers, and even airliners). The issue of allegiance was commented on by a member of a global automotive platform team:

"There are those in the platform project who take responsibility for all brands, but there are also those who want to transfer [responsibilities] to the other brands. It is easy to favour the own brand... you are affected by your environment. You should be 'genderless' when working for the platform. Most of the people working with the platform check things with their own brand first, perhaps because of the contacts that they have at their own brand and because of the lack of contacts at other platform brands."

When it comes to creativity in these kind of virtual teams, the conditions are more than a little constraining because team members always have to think long and hard about the downstream effects of 'creative propositions'. It can, for example, be extremely difficult to get acceptance for a creative idea that contributes to a cheaper platform, but which at the same time requires serious investments at another of the participating brands. An obvious reason for mistrust, and the essence of the trust dilemma, is that it is very difficult to make judgments about where collaborators' loyalty lies under various circumstances. For example, one person might be completely trusted when it comes to making platform decisions with minimum financial impact on her/his own brand, but when the stakes are higher, the same person might not be trusted since there is a risk that she/he will prioritise the brand before the platform. In one of the global automotive platform development projects, a team member noted:

"[They] want us to release our material five weeks earlier than we do now, and they will get results and details from tools fifteen weeks later than we would have got...it's a difference of twenty weeks. It's not logical. We should have the same 
amount of time... [They] have just looked out for their own interests, that we have a joint platform seems to be a minor detail here."

Since trust in virtual teams is very much based on the knowledge that persons have of each other, the work they do, and the work they have done in the past, it seems absolutely crucial to achieve a greater sense of social connectedness in virtual teams. Gaining a better understanding other people's priorities and problems, is highly important since it fundamentally sets the context for your own work. Observations have shown that engineers very often turn to a person for information rather than to a database or a file cabinet, and people seem to rely heavily on colleagues they know and trust. This raises questions of how to provide enough 'social lubrication' in virtual teams. Although permanent virtual teams are becoming increasingly common, many Virtual Enterprises face a reality where teams are formed and dissolved rapidly, and there is not a lot of time to make these teams 'gel'. Furthermore, there is a steady influx of temporary team members, consultants, experts etcetera, and they must also be natural partners in the strive for 'team-level creative synergy' (Kurtzberg \& Amabile, 2001). To recap, trust is contextual. It varies from situation to situation, and you trust people for different reasons. In the industry context we describe, it is dangerous to assume that trust has automatically been created just because the contracts have been signed, or that trust exists just because the appropriate security strategies have been put in place when it comes to data and information sharing over networks. For virtual teams that aim to be truly creative, trust can not be assumed, it has to be collectively created.

\section{Dilemma II: Shared Corporate Culture}

The dilemma of shared corporate culture is that when combining different corporate cultures, which is essentially what Virtual Enterprises are about, you are in effect creating a new culture. There are a lot of practical problems in this respect, some of which relate to the kinds of cultural traits that are transferred from the individual companies to the common Virtual Enterprise culture. Here, we are talking mainly about culture-as-practice, meaning 'the ways we do things around here'; ways of working that have evolved through the years and which are so deeply rooted in each company that they are more or less taken for granted.

Since an automotive platform development project is about joint commitments, which is also true with respect to joint life cycle commitments in the aerospace industry, we cannot assume that a shared culture already exists just because the paperwork has been signed. It is appealing to think about virtual teams creating 'virtual cultures', without necessarily having to change the internal corporate cultures that have been successful in the past. For example, we can speculate that the 'Toyota Way' (Liker, 2004) is based on a set of beliefs, values and principles that are not easily transferred into a Swedish business context, just in the same way as the 'Volvo Way' relies on approaches that might create havoc in other circumstances (even if applied to other Swedish companies). One Swedish automotive engineer with experience from global development had the following to say about differences in corporate culture:

"The Swedish style is open dialogue, no prestige... as a person you can say 'I think like this'. The Japanese style seems to be that you say something in the name of [the brand]. In the US, everything seems to be more individually oriented, but much more hierarchical than in Sweden."

Another Swedish automotive engineer further commented on pros and cons of various cultures: 
"When we were at [a company] in the US, two researchers from a university participated. Their first question after the meeting was 'who of you is the boss?' From the discussion, they couldn't understand who was the boss. We had five or seven people there and everyone was talking. ...but sometimes it seems like we are not as well aligned as [they] are. It is not good that we sometimes take a discussion with our boss during a meeting. [They] seem to have gone through such things beforehand, they are better prepared."

On a practical level, corporate culture impacts which things you prioritise. As in the section on trust, particular ways of working are sometimes viewed with suspicion from the perspective of other organisations, maybe because these approaches differ from what people in other organisations usually expect. For example, an automotive engineer working in global platform projects experienced differences in the way that stage gates were understood:

"We have not cleared cost and weight for the next gate. For [them] it is crucial that we're clear until then. We see it more as a way of highlighting problems at the gate...we tell it like it is and that is not always popular...there are often a lot of risks."

Similar observations were made by an aerospace engineer working in global partnerships:

"My feeling is that we are very honest. We flag when we have a problem, while others hide them. But that's just our point of view, it's an appraisal. Other people make other judgments. We wave our flag...now we have trouble...very early. Not the smartest way when the project is running since it puts us in the spotlight...but after the project we probably have a good result, while others might come up with late problems."

The engineers that we talked to seem to be very aware of differences in culture, and they are often quick to point out that their remote colleagues in partner companies probably do have similar concerns. Again, this is not about defining best-practice and worst-practice; rather to highlight some of the barriers to a shared, 'creative' culture. It is also a highly political game, where participants can use other organisations' cultural traits (e.g. work methods and principles) to their own advantage. When doing risk analysis, for example, 'waving the flag' or 'reporting red' can also be a conscious choice rather than simply a forced decision that attracts unwanted attention:

"As a project leader, you can be sly and put in a red marker...then you will come up high in the organisation and say 'we need more resources to reach our goals'. So, you can use that tool the other way around. Sometimes you flag red and end up in an undesired situation, sometimes you can get the things you want. That you've flagged red once in a while is forgotten in the end if you deliver what is expected."

In the case of automotive platform development, one of the project leaders commented on the importance of doing things in ways that are 'recognisable' to the partner's corporate culture, to increase the acceptance rate of their work outside of the internal organisation:

"[They] have a certain way of doing things. If they recognise things, it is easier for them to accept something. It is not only about documentation, but also about 
the way it is documented and presented. [They] often see problems in our presentations; they do not see it as feasible solutions. They are hung up on things that haven't been done. We should have followed [their] way of working from the start...I guess we wanted to keep [our] way, and I think that's one of the biggest problems."

Another interesting aspect that relates to shared culture, and how it impacts virtual teams and their creative boundaries, has to do with industry culture. Taking the aerospace industry as an example, there are very special demands that seriously affect a virtual development team's degree-of-freedom. There are regulations and contracts that govern what a system or a component must accomplish. To a certain extent, there are also regulations for what the processes (e.g. test routines) that lead to a finished product should look like. There is a striking difference between this reality and that of industries where you can repair faulty products after they have been released to customers, or those consumer-based industries where the customer might simply decide not to buy the product because it does not meet their demands.

What does this mean for virtual team creativity? On one hand, product failures could result in massive and hugely expensive recalls (such as in the automotive industry). On the other hand, product failures could also put lives at danger, summarised here by Volvo Aero Corporation's former President and CEO, Fred Bodin:

"As far as quality is concerned Volvo Aero must be in a class of its own. In the aviation industry quality and safety go hand-in-hand. A car can be recalled, but in aviation everything has to be right the first time." (Volvo Aero Corporation, 2005, p. 14)

Kurtzberg \& Amabile (2001) note that diversity among team members can be both positive (i.e. presenting a heterogeneous set of perspectives for consideration) and negative (limiting shared understanding by creating such divergence that damaging conflict occurs). In this light, we think that Hirshberg's (1999, p. 33) concept of 'creative abrasion' is very appealing. It deals with the need to handle colliding cultures and conflicting viewpoints "without discarding or allowing either to dominate". Creative abrasion recognizes that friction has positive properties that are crucial in making things work (e.g. friction as a source of electricity), and that we need to better understand when these abrasions have creative potential, and when they have not. As we see it, it could be useful to think in terms of creating 'third cultures' (Graen \& Wakabayashi, 1994) - a way of working in global teams that is not necessarily based on co-located models of collaboration, or based on a single dominating worldview. Instead of saying that streamlined development is not favourable to creative activity, we might try to identify the positive abrasions between corporate cultures that can help spur creativity, and try to better understand the ways in which the nature of streamlined industries actually empowers, rather than limits, our creative potential.

\section{Dilemma III: Shared Goals}

The dilemma of shared goals is that in order to fulfil them you often have to take on individual, team or organisational commitments and responsibilities that could involve conflicting sub-goals. From a Virtual Enterprise perspective this relates closely to our previous discussions about both trust and culture: of course these concepts are enmeshed in each other in that they are all about accomplishing a sense of shared understanding of the project, the roles of the participants, and the expectations on what every partner should bring 
to the table to fulfil the joint contractual agreement. It is important to discuss them separately as they affect creativity in different ways.

In our discussions with automotive engineers, they often talk about 'finding the best compromise'. Everything you do to optimise a particular component might have serious negative effects on other components or on the system as a whole. So, even if you are able to find a 'creative solution', you are soon under pressure to explain how your solution influences the commonality (which in turn has to do with economies of scale), and how your idea relates to the manufacturing tools that are currently available at the plants etcetera. Also, as we touched on earlier, you could come up with a brilliant solution that is of great benefit to all platform partners (e.g. increased commonality with improved performance), but which could at the same time result in major and expensive upgrades to your own part of the platform. Here, it is easy to understand that low-level actions (such as making a minor design change) could bring about consequences of unforeseen proportions - an incentive as good as any for staying 'inside-the-box'. One of the automotive engineers we have talked to says the following about shared goals in platform development:

"Each brand wants to have products that are profitable. If Volvo makes a profit, it goes into Ford, but a certain amount goes back to Volvo for the next model. [Another brand] is completely dependent on [their automotive group], so they can't do any development on their own. Shared goals could mean making a profit in order to keep your freedom."

From an aero engine development perspective, a project leader commented on the difficulty of knowing whether or not the distributed team really have shared goals and priorities:

"I have probably not really understood what the others think is important.

Priority one is to deliver, but after that... You would think that you could read it in the contract... [Engine manufacturer] and [airline] might not agree with each other, but we haven't talked to [the airline] that many times. It's difficult to get the whole picture...some of the things are kept secret. Their problems might be based in earlier engine programs and business relations."

It is rather obvious that engineers working in such global, cross-boundary alliances constantly have to deal with conflicting demands. It is not simply the case of sitting down at a table and definitively deciding on shared goals and objectives. Rather, they have to move between different levels of abstraction in that some goals are of superior importance in particular circumstances, but of inferior importance in others. High-level objectives, such as meeting new flight regulations concerning noise, must be assessed in relation to lower-level objectives, such as making strength simulations of a modified engine casing to allow for noise reduction. Also, the engine manufacturer (e.g. Rolls-Royce) have separate contractual agreements with the aircraft manufacturer (e.g. Airbus), and this information is mostly not available to the component manufacturer (e.g. Volvo Aero). A Volvo Aero project leader comments on the difficulties of relating their own goals, based on written contracts, to the higher level goals that might be part of undisclosed business agreements between the other partners:

“We must hope that they've written clear enough contracts with us...requirements specifications, penalties etcetera. We have demands to deliver on time, at a certain weight, but we don't know what the penalty structure between [engine 
manufacturer] and [aircraft manufacturer] looks like. [The engine manufacturer] might choose between a weight penalty and a delay penalty, and decide that it's better to deliver late. If they don't feed this down to us, we have a hard time seeing the type of trade-offs we do at our level. And even if we meet our demands, [the engine manufacturer] might have other problems that result in penalties. We only get a small piece of the puzzle."

Apart from contractual obligations, there are flight safety regulations that impact how companies work and how they document their work to ensure traceability. Further, an individual aero engine component has clear interfaces to other components that make up the engine, so the geometrical 'envelope' together with the very high performance requirements means that the creative boundaries have, at least to a great extent, already been set. Of course, this is also true in automotive development, where engine envelopes, foot envelopes, wheel envelopes etcetera govern the geometrical boundaries (i.e. 'vehicle packaging'). Just as all components need to fit into the car and contribute to the overall performance, the aero engine is also a system that must work together with the airplane. All parts must contribute to the overall system performance and we must therefore consider that a modification requiring 1000 hours of work on the system level might render 3000 hours for every component in that system. Whose goals are really targeted, who gets the benefits of reaching those targets, and who is paying for the work to reach them? A Volvo Aero project leader highlighted this problematic situation:

"[The engine manufacturer] owns the engine certificate, so their perspective is what's best for the engine. If we and [our competitor] stand there, [the chief engineer] will choose what is best for the engine, that's what makes the engine sell. But if you are at a crossroads...we are partners and carry the costs... [the chief engineer] doesn't consider our economical aspects, but will take what is best for the engine. My gut feeling tells me that they look more carefully when they're paying for things themselves. We are also thinking 'Volvo Aero'....we can have our own purchasing strategies... We are working for Volvo Aero; we must have our own interests."

When discussing shared goals we are really discussing 'collective commitment'. What are the goals that we all are committed to achieving, and what are the goals that only apply to some of us? This relates to how we define our virtual teams: are we considered a team just because we are all engineers, are we considered a team because we are tied together in the organisational chart, or are we considered a team because we have joint commitments that we achieve together rather than individually? Sometimes it is obvious that team members have the same perception of the goal, but radically different views on how to reach that goal.

We believe that the notion of a virtual team, at least in streamlined development, involves often conflicting worldviews, approaches and priorities. Again, the challenge is not necessarily to completely merge these different perspectives, but to raise awareness of their complementarynature, and to help virtual teams know when they have to collaborate, and when they might be better off on their own. Being able to effectively identify critical collaborative activities, or 'interfaces' between individuals and teams, might be more interesting than always striving for close collaboration. 


\section{Dilemma IV: Creative Priority}

The dilemma of the creative priority is that not investing in creativity could in fact increase the creative output. What we are saying here is not that companies should refrain from dedicating extra time, money and resources into creativity-enhancing approaches. Rather streamlined development means that those extra resources are often not available, and furthermore, the immense pressures of such industries could actually prove to be a driver of creativity and innovation. In the words of an aero engine project leader: "He who suffers conquers".

This comment should be seen as a general comment on the current incentives for creativity and collaboration that are available to engineers working in global product development of the Virtual Enterprise kind. Relating to the introduction of this chapter, where we talked about 'creative work' versus 'routine work', we could ask ourselves if more time and money automatically results in more creative work, or if "work expands so as to fill the time available for its completion", as Parkinson's Law (Parkinson, 1958) states. What does it mean to put a priority on creativity? Will people be more creative just because they have more time, or could it be that creativity is needed the most, and is also most applicable, when enmeshed in daily, supposedly 'routine' work, such as designing aero engine components or automotive platform components as part of highly streamlined and regulated processes? As one of the automotive engineers put it:

"When should you be creative? You can be creative and cut costs. When can you afford to be creative, and when can you not afford to be creative? ...creativity can be to do the same thing cheaper. Creativity can be to cut costs with sustained or increased performance. Cars get cheaper every year... we have to try to lower the price without making the product worse."

Another automotive engineer had similar thoughts:

"We have to be creative to put together a product where several brands take part and we have different production plants. The biggest challenge is the complexity...very many boundaries are set with respect to five production plants."

When we have talked to engineers working with streamlined development, we have seldom heard them talking about creativity, at least not in those terms, until we have explicitly asked how they relate to creativity. Part of this could imply that they do not really consider 'streamlining' to involve creative activities. It could also be that they are not that concerned if other people consider their work to be creative or not. They are working towards meeting deadlines, satisfying requirements specifications, fulfilling contract agreements - they are doing what they have to, solving problems as they arise. One aero engine project leader summarised the demands on his team in the following way: "The biggest problem of all is to deliver on time".

Investing in creativity is truly a hard sell considering the lead times that development teams face. From one project to the next, Volvo Aero cut their lead time (design to delivery) from 19 months to 12 months, which caused one of the engineers to comment: "Innovation disappears when the contract is written. After that, it's only about bringing the stuff out". 
One of his colleagues, a project leader, added to this comment:

"We have very little time for innovation... we have to go for safe solutions. We have to know that what we're doing works to ninety-five percent. You glance a lot at how earlier generations look...we go for the things that give us a competitive advantage... and with low risk. No customer wants us to take big risks."

In a way, our observations and experiences tell us that creativity flourishes under pressure. Creativity inside-the-box is entirely possible; otherwise these companies would not be able to deliver innovations to the market. Considering the creative boundaries that are inherent in streamlined industries, it is amazing that creative activities do not cease to exist. One of the reasons for this might be that the 'not invented here' syndrome is not given enough time (or funds) to develop. New work methods and design solutions seem to gain acceptance more easily when teams are under pressure, perhaps because stakeholders are forced to find the 'best argument' or 'best compromise', regardless of the origin of the idea. Interestingly, this implies that although the high industry demands and complex business relationships provide a rather narrow window of opportunity for creative freedom, it is these very same constraints that encourage participants to really collaborate, find shared understanding, and reach acceptable compromises that 'do the job'.

When seeing the pressures of streamlined development not as something entirely negative, but as a sometimes crucial component of creative work, we can make the analogy with athletes that get into 'game mode' by, more or less consciously, using their pre-game stress to stimulate the flow of adrenaline into the bloodstream. One of the problems here is that virtual teams often seem to play on different battlefields. When the adrenaline is pumping in Detroit due to near-deadline design changes, the people in Gothenburg might be out playing golf, and vice versa. In discussions with aero engineers, for example, they mentioned the problems of 'distributing heat', meaning the possibility to make off-site team members aware of the severity of a particular situation.

\section{Future Trends}

One of the key messages that we take from the battlefield tales above is that the applicability of information and knowledge is a continuously moving target. The problem you start out working on is often not the same problem a few weeks down the line, and the solutions you discarded in previous projects might very well prove to be useful in future projects, due to rapid and radical advances in both supporting methods and technologies. Furthermore, the agility required of future enterprises forces them to quickly decide when collaboration is beneficial, when it is too risky, and on what level of interaction that collaboration should best be carried out. In many previous cases, the focus has been directed on the technical challenges that virtual teams face, while it has been repeatedly discovered that communicative aspects have a decisive impact on the success of the team performance (i.e. how well virtual teams actually come to understand and jointly deal with those technical challenges).

The status, applicability and validity of dispersed knowledge resources needs to be continuously assessed, updated and effectively spread to all stakeholders in a project to ensure that no time, money or intellectual resources are lost on activities that are based on inaccurate (or irrelevant) information. Here, we are basically talking about knowledge efficiency, where the competitive edge lies in a company's ability to pick out the useful knowledge, while leaving out the irrelevant, and to take advantage of that useful knowledge quicker than their competitors. Using an organic analogy, we need to aim for a high 'knowledge metabolism'. 
Being in possession of information and knowledge is obviously crucial, but putting it to use, in the right context, at the right time is essentially what decides if it is the 'right' information and knowledge or not. Information and knowledge thus needs to be shared on all levels of maturity - from sketchy ideas to minutely detailed contracts - so that useful ideas and concepts are shared when needed rather than when defined in the stage gate process. Today, such informal knowledge sharing is extremely difficult to achieve across geographical, functional, and organisational boundaries. This has to change. Here, it is of great interest to explore the concept of 'Knowledge Readiness Level' (KRL), brought forward by NASA along the same lines as their 'Technology Readiness Level' (TRL) concept (Chiaramonte \& Joshi, 2004). Future KRL systems could help virtual teams assess both the current status of their knowledge capability and devise strategies to reach a satisfactory level.

Future Enterprise environments will be characterized by multi-context collaboration, where people will need to move seamlessly between different teams, projects and priorities. They will need to switch effortlessly between total task-focus and multi-tasking, between high-level goals and low-level goals, and between individual work and exceptionally close teamwork. Further, people will have to move fluently between both virtual and physical team spaces, all the time with uninterrupted access to colleagues, information systems and other knowledge resources that are spread all over the world. Taken together, this is pointing towards an anyplace/anytime vision of collaborative engineering, where virtual teams are so commonly used that it will be very difficult to draw the borders between the co-located and the virtual workplace. Most likely, even physically co-located team members will communicate to a greater extent in a virtual mode, enabling all virtual team members to more easily stay aware of what is going on at partner sites. The transparency and seamlessness of collaboration implies that engineers will be allowed to focus more closely on the job of work (i.e. delivering the goods) and that they, in doing that job, will be less bound to physical spaces and less dependent on the immediate availability of their co-located colleagues.

This multi-context perspective on collaboration further implies a need for automated capabilities to link virtual team members to relevant information and knowledge, reducing the necessity to clearly know what you are looking for. Further,it is unlikely that Future Enterprises will be able to maintain their agility if they are constantly forced to homogenize the IS/IT environment to include all partner's needs and requirements. Instead, there is a clear trend towards supporting heterogeneous enterprises with service-oriented architectures, allowing companies to share data, information and knowledge regardless of which vendors and systems have been selected at each partner site. The 'Virtual Enterprise Collaboration Hub', currently under development in the EU FP6 aeronautics project VIVACE (2005), provides one example of how such a neutral collaboration platform could be realised. So, even though we can see a convergence in terms of the capabilities that virtual teams would need to succeed in global collaboration, such as having one-click access to both audio, video and web conferencing, it does not imply that these capabilities should be delivered by one and the same vendor. Rather, there is going to be an increasingly open 'knowledge marketplace' where service providers of all sizes will be able to contribute with, for example, simulation capabilities that can easily be incorporated into a partner's workflow without exposing any confidential information ('black box' approach with internal system properties hidden from view).

Best practice until quite recently for teamwork in engineering has been to rely on physical colocation for intense collaboration. As a consequence of escalating globalisation, development is now conducted worldwide in highly heterogenous teams, which makes physical co-location 
extremely difficult. In relation to the industry contexts we have discussed earlier, we can see the following general trend in virtual team collaboration (although there are many dimensions that we do not consider in Table 2):

\begin{tabular}{|c|c|c|}
\hline As-Was & As-Is & To-Be \\
\hline $\begin{array}{l}\text { Most team members located at } \\
\text { partner site, with back-office } \\
\text { support. }\end{array}$ & $\begin{array}{l}\text { Virtual teams with some on-site } \\
\text { ('coordinator') representation. }\end{array}$ & $\begin{array}{l}\text { Completely virtual teams. No-on site } \\
\text { representation. Even co-located } \\
\text { teams are 'virtual'. }\end{array}$ \\
\hline $\begin{array}{l}\text { Global person-to-person } \\
\text { collaboration (with back-office) } \\
\text { occurs predominantly in formal } \\
\text { meetings. }\end{array}$ & $\begin{array}{l}\text { Global person-to-person } \\
\text { collaboration occurs } \\
\text { predominantly in formal } \\
\text { meetings. }\end{array}$ & $\begin{array}{l}\text { Global person-to-person } \\
\text { collaboration occurs } 24 / 7 \text { in varying } \\
\text { degree of formality. }\end{array}$ \\
\hline $\begin{array}{l}\text { Several employees are detached } \\
\text { from their mother company. }\end{array}$ & $\begin{array}{l}\text { Few employees are detached } \\
\text { from their mother company. }\end{array}$ & $\begin{array}{l}\text { No employees are detached from } \\
\text { their mother company. }\end{array}$ \\
\hline $\begin{array}{l}\text { Several employees are dedicated to } \\
\text { single projects. }\end{array}$ & $\begin{array}{l}\text { Few employees are dedicated to } \\
\text { single projects. }\end{array}$ & $\begin{array}{l}\text { No employees are dedicated to } \\
\text { single projects. }\end{array}$ \\
\hline $\begin{array}{l}\text { On-site team members are } \\
\text { disproportionately affected by } \\
\text { pressure from the partner company. }\end{array}$ & $\begin{array}{l}\text { Coordinators are } \\
\text { disproportionately affected by } \\
\text { pressure from the partner } \\
\text { company. }\end{array}$ & Pressure is evenly distributed. \\
\hline $\begin{array}{l}\text { High social connectedness with co- } \\
\text { located team members (at partner } \\
\text { site). Low social connectedness with } \\
\text { back-office members (at mother } \\
\text { company). }\end{array}$ & $\begin{array}{l}\text { High social connectedness with } \\
\text { co-located team members. Low } \\
\text { social connectedness with virtual } \\
\text { team members. }\end{array}$ & $\begin{array}{l}\text { Social connectedness is less } \\
\text { dependent on physical proximity. }\end{array}$ \\
\hline $\begin{array}{l}\text { Culture assimilation: 'guests' are } \\
\text { expected to follow the work } \\
\text { approach governed on-site. }\end{array}$ & $\begin{array}{l}\text { Culture dominance: work } \\
\text { approach is dominated by 'lead' } \\
\text { partner. }\end{array}$ & $\begin{array}{l}\text { Third culture: work approach is } \\
\text { sensitive to the interests of all } \\
\text { partners. }\end{array}$ \\
\hline
\end{tabular}

Table 2. Towards Future Enterprise Collaboration.

\section{Conclusions}

This chapter uses observations and experiences from real industry practice to point out that we might be in need of a more balanced view on creativity's role in virtual team collaboration - particularly when applied to so called streamlined development. Although we are generally positive to the quest for 'creativity techniques', we are also painfully aware of the severe constraints that currently impair creativity. Furthermore, we firmly believe that many of these constraints are here to stay and that they are going to be even more impeding in the future. This means that we should not assume that companies will 'get the point' and start investing heavily in creativity and in 'creativity enabling' activities. The fact is, many companies have already done so - with poor resulting ROI. What has been gained in one dimension (e.g. social connectedness) has often been lost in another (e.g. effectiveness), and this is partly because these 'investments' seem to require substantial downpayments in terms of each team member's time and devotion. This is a major flaw.

We need to build social connectedness as a natural part of day-to-day work, we need to create trust through everyday interactions between virtual team members, we need to encourage creative work in just about everything we do in the workplace - but we simply have to understand that when we focus explicitly on 'creativity' or 'trust', or any other concept, we naturally lose some of our main focus, to 'deliver what has been promised'. Virtual teams that will be able to build the foundation for creativity in natural, seamless, and effortless ways will be way ahead of their competition. 
On a positive note, there are already trends that point towards a business environment where the open source and standards movement will play an increasingly important role. In terms of virtual teams and creativity, this movement is acknowledging that highly heterogeneous enterprises must be given a 'common playground' where true, hugely creative collaboration can take place in spite of the rich diversity of backgrounds, cultures, organisational and technical setups etcetera that the various partners embrace. If we can create this common playground, we might more truthfully say that we have reached success 'because of' diversity, rather than 'in spite of' diversity. Furthermore, in the spirit of true collaboration and joint creativity, future books on the subject might be drawing on 'tales from the playground' rather than 'tales from the battlefield'.

\section{Author Information}

Dr. Andreas Larsson, Assistant Professor, Division of Computer Aided Design, Luleå University of Technology, Luleå, Sweden. E-mail: Andreas.C.Larsson@ltu.se, Phone: +46920 491874, URL: http://www.cad.ltu.se

Dr. Tobias Larsson, Associate Professor, Division of Computer Aided Design, Luleå University of Technology, Luleå, Sweden. E-mail: Tobias.C.Larsson@1tu.se, Phone: +46920 493043, URL: http://www.cad.ltu.se

Dr. Nicklas Bylund, Volvo Car Corporation, Gothenburg, Sweden. Resident Engineer at Ford of Europe, Cologne, Germany. E-mail: nicklasbylund@hotmail.com, Phone: +46 (0)702479801

Dr. Ola Isaksson, Company Specialist in Engineering Design, Volvo Aero Corporation, Trollhättan, Sweden. E-mail: Ola.Isaksson@volvo.com, Phone: +46 520 93987, URL: http://www.volvo.com

\section{References}

Brandenburger, A. M., \& Nalebuff, B. J. (1997). Co-Opetition: A Revolution Mindset That Combines Competition and Cooperation: The Game Theory Strategy That's Changing the Game of Business. New York, NY: Doubleday/Currency.

Byrne, J. (1993). The Virtual Corporation. Business Week. February 8.

Chiaramonte, F. P., \& Joshi, J. A. (2004). Workshop on Critical Issues in Microgravity, Fluids, Transport, and Reaction Processes in Advanced Human Support Technology. NASA/TM-2004-212940. Retrieved October 30, 2006, from http://peer1.nasaprs.com/peer_review/prog/ahst_2003.pdf

Davidow, W. \& Malone, M. (1992). The Virtual Corporation. New York, NY: HarperCollins.

Graen, G. B., \& Wakabayashi, M. (1994). Cross-cultural Leadership-making: Bridging American \& Japanese diversity for team advantage. In H. C. Triandis, M. D. Dunnette, \& L. M. Hough (Eds.), Handbook of industrial and organizational psychology, 4, 415-446. New York, NY: Consulting Psychologist Press.

Hirshberg, J. (1999). The Creative Priority. New York, NY: HarperCollins.

Kurtzberg, T. R., \& Amabile, T. M. (2001). From Guilford to Creative Synergy: Opening the Black Box of Team-level Creativity. Creativity Research Journal, 13(3\&4), 285-294.

Liker, J. (2004). The Toyota Way. New York, NY: McGraw-Hill. 
Parkinson, C. N. (1958). Parkinson's Law: The Pursuit of Progress. London: John Murray.

Sutton, R. I. (2001). Weird Ideas that Work: 11 1/2 Practices for Promoting, Managing, and Sustaining Innovation. New York, NY: The Free Press.

VIVACE Project. (2005). Virtual Enterprise Collaboration Hub - Services Description. Retrieved October 30, 2006, from http://www.vivaceproject.com/content/advanced/vechsd_full.pdf

Volvo Aero Corporation. (2005). AERO: News, Views \& Interviews. No 3.

WebEx Communications, Inc. (2006). Web Conferencing, Online Meetings, and Video Conferencing. Retrieved April 30, 2006, from www.webex.com 